\title{
THE PHYSICAL EVALUATION OF SEISMIC DESTRUCTIVENESS*
}

\section{By Hugo Beniof}

The problem of designing structures to withstand destructive earthquakes is not in a very satisfactory condition. On the one hand engineers do not know what characteristics of the ground motion are responsible for destruction, and on the other hand seismologists have no measurements of seismic motion which are sufficiently adequate to serve for design, even if the destructive characteristics were known. Consequently, engineers have been forced to proceed on an empirical basis. From past experience, chiefly in Japan, it has been found that buildings which are designed to withstand a constant horizontal acceleration of 0.1 gravity are, on the whole, fairly resistant to seismic damage. It is fortunate that such a simple formula works at all, in view of its inadequacy from the point of view of precise computation. We know that seismic motions do not exhibit constant accelerations; that instead they are made up of exceedingly variable oscillatory movements. A formula based upon constant acceleration may thus lead to large errors, especially when applied to new types of structures which have not been tested in actual earthquakes. In the following paragraphs a new formula for seismic destructiveness is proposed, in the belief that it is more accurate than previous ones. In addition to providing engineers with a more rational basis for design procedure, it determines a new type of seismographic instrument for recording and measuring the destructive characteristics of seismic motion.

The actual destructiveness of a given earthquake might be defined as the sum of all the material damage caused by the seismic movements. Such a definition does not lend itself to physical measurement and is therefore unsatisfactory from the physical point of view. The idea contained in this definition may be extended to permit physical measurements. Thus, if strain gauges were erected on all members of all the involved structures, the measure of seismic destructiveness could be taken as the sum of all the indicated strains. These definitions of destructive-

* [Received for publication May 26, 1934.] 
ness, though ideal from the point of view of completeness, are impracticable to carry out. They do suggest, however, a formula having a high degree of practicability. Thus, suppose we substitute for the engineering structures a series of undamped pendulum, seismometers having frequencies ranging from the lowest fundamental frequency of engineering structures to the highest significant overtones. During an earthquake each component seismometer would write a characteristic seismogram. Plotting the maximum recorded deflection of each pendulum against its frequency, we obtain a curve which may be termed the undamped pendular spectrum of the earthquake (see Fig. 1). We now define seismic

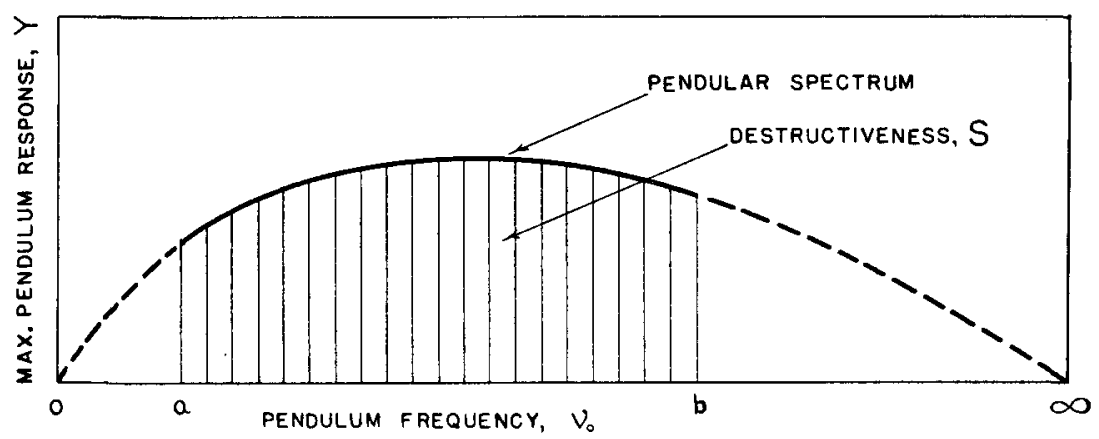

FIG. 1.-Pendular spectrum and seismic destructiveness

destructiveness as the area between this curve and the axis of abscissas. More precisely, seismic destructiveness is the integral with respect to pendulum frequency of the maximum displacement of an infinite series of undamped pendulums extending over the significant range of frequencies. Since the duration of violent movement is in general short, there will be no narrow humps in the pendular spectrum and consequently the integral will be closely approximated by the polygon given by the maximum deflections of a small finite number of pendulums. Probably twenty will be sufficient.

The application of this formula to design is straightforward. We shall first consider a pendular structure such as a water tower, in which the mass is effectively concentrated in the tank and the restoring force is concentrated in the supporting members. Such a structure responds to seismic movements substantially as a simple pendulum seismometer. Hence, if its frequency is known, the maximum displacement of its center of oscillation is given by the observed amplitude of the pendular spectrum at the same frequency. With this displacement given, all of the resulting 
structural strains may be readily calculated. Thus, with this formula the problem of design is reduced to the computation, or measurement, of the vibration frequencies of the structure.

Complex structures having more than one mode of vibration may be considered as made up of a group of pendulums with the frequencies of the individual modes. The resulting maximum displacements are given by the sum of -the individual displacements, due regard being given, of course, to the existence of nodes and loops. In the case of structures which may be approximated by rods vibrating transversally, the natural frequencies and strains can be calculated by the formulas given by Le Conte and Younger. ${ }^{1}$ The more complex structures are now being studied experimentally, or theoretically, by a number of investigators and it is to be expected that their results will be of great value in the application of the pendular spectrum method.

In the preceding discussion, structural damping has been neglected. In general, the errors introduced by this simplification are quite small. Thus, for example, we may consider the effects of damping on a single vibration mode of a structure. When subjected to a resonant vibratory force, it responds with an amplitude which increases asymptotically with the time to a maximum value determined by the damping constant. The time constant, defined as the time for the amplitude to rise to $1-\frac{1}{e}$ of its final value, varies inversely with the damping constant. It is clear that if the resonant force acts for a time interval which is short compared to the time constant of the system, the resulting amplitude is independent of the damping. Now in the case of most engineering structures the damping is small. Furthermore, the time interval during which a finite resonant force may be effective in an earthquake, though not known precisely, must be quite short. Consequently we may expect the structural vibration amplitudes to be substantially independent of the damping. At any rate, calculations based upon the undamped pendular spectrum determine the upper limit for strains in any structure.

Whenever greater precision is required, it will be necessary to set up an additional series of critically damped pendulums for recording the critically damped pendular spectrum. This damped spectrum determines the lower limit of strains, since, in general, the damping constants of structures lie between zero and the critical value. With the two spectra

${ }^{1}$ Joseph N. Le Conte and John E. Younger, "Stresses in a Vertical Elastic Rod when Subjected to a Harmonic Motion of One End," Bulletin of the Seismological Society of America, 22, 1, March, 1932. 
given, the strains can be computed with engineering accuracy for any value of structural damping. Observations with a set of seismographs designed by the writer for use by Professor Martel, of the California Institute of Technology, have shown that the tops of buildings exhibit vibration amplitudes of approximately three times those of the ground at the bases. On the basis of these experiments we may expect the undamped pendular spectrum to have amplitudes approximately three times those of the corresponding damped spectrum.

It should be emphasized that the formula of destructiveness defined in this paper refers solely to elastic deformations in which stress and strain are proportionate. In the event of actual destruction, elastic limits are exceeded and in this condition strains cannot be accurately calculated by this method. This limitation is of no serious consequence, since it is customary in good engineering practice to keep strains within the elastic limit.

It might be well to consider some of the characteristics of seismic pendular spectra which may be predicted on the basis of present knowledge. In the case of very small earthquakes, observations at a given point should indicate very nearly identical spectra for all shocks originating at a common focus. Larger shocks with a common focus may be expected to show greater spectral differences. In addition, the spectral energy of large earthquakes should be shifted toward the low frequencies, as compared with smaller shocks. The spectrum of a given earthquake changes from point to point, depending upon distance from the origin and upon ground characteristics. Increasing distance from the focus shifts the spectral energy toward the lower frequencies. A free vibration of the ground will be indicated by a sharp maximum in the undamped spectrum, which does not appear in the damped spectrum. This will be true regardless of whether the free vibration exists in the region of the focus or in the region of the observing station.

To be most useful, observations on pendular spectra should be maintained throughout populated regions for a long enough period to record strong earthquakes from all active foci. The resulting records of seismic spectra would then be sufficiently complete for all future engineering purposes. It may be, however, that a much less ambitious program would suffice. Thus, for example, a few observations on strong earthquakes may demonstrate a sufficient similarity in their spectra to warrant the practical use of a single generalized spectrum for all foci. Variations would be necessary, of course, to allow for differences in ground conditions at the building site. Furthermore, we may find that spectra of large 
and small earthquakes with a common focus are regularly related in such a. way that the spectrum of a large earthquake may be easily computed from that of a corresponding small shock. If this be true, the time required for an adequate series of observations on the spectra of a region will be greatly reduced.

The spectral formula for seismic destructiveness has been developed in the preceding paragraphs on the basis of an intuitional argument. Originally, however, it was derived from a fundamental physical concept which will now be indicated briefly.

A wave disturbance $f(t)$, existing only in the finite interval $0 \leqq t \leqq T$, is represented analytically by the Fourier Integral ${ }^{2}$

in which

$$
f(t)=2 \int_{0}^{\infty} F(v) \cos [2 \pi v t+\vartheta(2 \pi v t)] d v
$$

$$
[F(v)]^{2}=\left[\int_{0}^{T} f(t) \cos 2 \pi v t d t\right]^{2}+\left[\int_{0}^{T} f(t) \sin 2 \pi v t d t\right]^{2}
$$

The significance of this Fourier Integral lies in the fact that it represents the sum of an infinite number of simple harmonic components of frequencies $v$ extending from zero to infinity, with definite phase angles $\boldsymbol{\vartheta}$. Furthermore, the amplitudes of the individual components are infinitesimally small. Thus, we see that the Fourier Integral (1) represents a continuous spectrum. The response of an undamped pendulum to a simple harmonic displacement of the ground is

in which

$$
y=\frac{v^{2}}{v_{0}^{2}-v^{2}} a \cos 2 \pi v(t+\delta)
$$

$$
\begin{aligned}
a & =\text { the maximum amplitude. } \\
v_{0} & =\text { the frequency of the pendulum. } \\
v & =\text { the frequency of the earth-wave. } \\
\delta & =\text { the phase angle of the earth-wave. }
\end{aligned}
$$

2 J. R. Carson and O. J. Zobel, "Transient Oscillations in Electric WaveFilters," The Bell System Technical Journal, 2, 1, July, 1923; and J. R. Carson, "Selective Circuits and Static Interference," ibid., 4, 268, April, 1925. Also M. Biot, "Theory of Elastic Systems Vibrating under Transient Impulse, with an Application to Earthquake Proof Buildings," Proceedings of the National Academy of Sciences, 19, 262-68, 1933; and M. Biot, "Acoustic Spectrum of an Elastic Body Submitted to a Shock," Journal of the Acoustic Society of America, 5, 206, January, 1934. 
From Equation 1 we find that a single component of the seismic disturbance is given by

$$
2 F(v) \cos [2 \pi v t+\vartheta(2 \pi v)] d v
$$

Hence, the response of a pendulum to a single component is

$$
\frac{2 v^{2}}{v_{0}{ }^{2}-v^{2}} F(v) \cos [2 \pi v t+\vartheta(2 \pi v)] d v
$$

and the response to the whole spectrum is

$$
y=2 \int_{0}^{\infty} \frac{v^{2}}{v_{u^{2}}{ }^{2}-v^{2}} F(v) \cos [2 \pi v t+\vartheta(2 \pi v t)] d v
$$

At some time during the passage of the seismic wave-train the response of the pendulum will indicate a maximum value, $Y$. Taking an infinite number of pendulums, the pendular spectrum is given by the function

$$
Y=\psi\left(v_{0}\right)
$$

and seismic destructiveness is defined by the integral,

$$
S=\int_{a}^{b} Y d v_{0}
$$

These are both shown in the diagram of Figure 1.

The limits $a$ and $b$ must be determined from observations on buildings. The integral $S$ defines a new intensity scale based upon physical measurements. For statistical study and comparison of earthquakes it should be much more accurate than the present scales based upon random observations. In effect it measures the potential destructiveness of a given earthquake to a standard city composed of simple standard structures.

From the engineering standpoint in the calculation and design of individual structures, the pendular spectrum, $Y$, is the function of primary interest, since it determines the structural response to earthquakes. The use of this function in the calculation of structural strains has the very great advantage that the true ground displacement (or its derivatives) does not have to be measured or calculated.

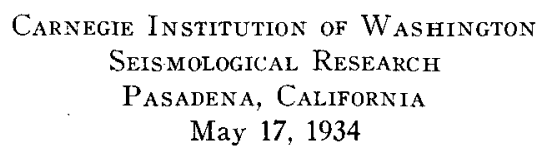

